\title{
Access and rational use of psychotropic medications in low- and middle-income countries
}

\author{
P. Padmanathan* and D. Rai \\ School of Social and Community Medicine, University of Bristol, Canynge Hall, 39 Whatley Road, Bristol BS8 2PS, UK
}

Improving access and rational use of psychotropic medications in low- and middle-income countries is an important factor in reducing the public health burden resulting from mental illness. This paper considers each component of the medications management cycle to identify current barriers to improvement. Selection is hindered by a lack of up to date local essential drugs lists, while procurement and distribution can be affected by the type of system used: centralised or decentralised, government-run or independent, push or pull. Rational use involves patients, prescribers and policy-makers and requires consideration of who is able to prescribe, how prescribing decisions are made and how to ensure patient-centred care. We include a number of recommendations based on these issues, while emphasising the importance of ensuring the broader context of mental illness and its management is not overlooked when improving access to psychotropic medications.

First published online 21 September 2015

Key words: Access, low-and middle-income countries, psychotropic medications, use.

\section{Introduction}

Mental and substance misuse disorders account for the greatest number of years lived with disability globally (Whiteford et al. 2013). In recent years, a growing evidence base has led to widespread recognition of these disorders as a significant public health issue in lowand middle-income countries (Patel, 2007a). The World Health Organisation (WHO) has highlighted the role for both psychosocial and pharmacological interventions in the management of mental illness (WHO, 2001, 2011a). In many low- and middle-income countries however, government-funded psychosocial interventions are almost non-existent and pharmaceutical therapies therefore often form the mainstay of treatment (Raja et al. 2015). Consequently, limited access to psychotropic medications may significantly contribute to the public health burden of untreated mental disorders. It is estimated that approximately four in five people in need of mental health or neurological care in low- and middle income countries do not receive it, and of those who do, the care received is often not evidence-based (WHO, 2011a).

Few would argue against the suggestion that health systems in low- and middle-income countries should aspire towards a comprehensive bio-psycho-social approach to the management of mental disorders (Engel, 1977). It is also notable that psychosocial as well as biological interventions have been demonstrated to

\footnotetext{
* Address for correspondence: P. Padmanathan, School of Social and Community Medicine, University of Bristol, Canynge Hall, 39 Whatley Road, Bristol BS8 2PS, UK.

(Email: prianka.padmanathan@gmail.com)
}

be effective in low- and middle-income countries (Patel et al. 2007b). Furthermore an over-reliance on medication prescribing may create a false economy; prescribing often requires access to more highly trained health workers which are a relatively expensive resource in short supply. There is also a danger that by solely improving access of psychotropic medications in areas where psychosocial interventions are unavailable, symptoms may be inappropriately medicalised and people with mental illness may be subjected to unnecessary side effects. This paper therefore focuses on recommendations for improving access to psychotropic medications strictly in the context of advocating for improved overall care for people with mental illness. This would preferably be in the form of a stepped care approach, in which only the more severe illnesses would require biological treatments.

There are various determinants of access, a term that embodies the concepts of 'availability' as well as 'affordability' (Raja et al. 2015). Although the private sector can often play a significant role in the provision of psychotropic medications, particularly when no alternative exists, we focus here on access and rational use via the public sector.

Access to all medications in the public sector remains limited (WHO, 2011b). Psychotropic medications however are subject to further challenges. The reputation of psychotropic medications as having a potential for dependence and abuse can lead to additional national regulation (WHO, 2005). Although this regulation may be necessary in certain circumstances, it should be noted that most medications for the treatment of common mental disorders have little 
dependence potential. The medications that do have properties that may create dependence, such as the benzodiazepines are not recommended as first line for any mental disorder (NICE, 2011). There is therefore a risk that excessive caution and stigmatisation may negatively impact on people receiving useful treatment.

Internationally, access can be limited via the Agreement on Trade-Related Aspects of Intellectual Property Rights, which introduced a 20 year patent protection for new medications. In order to allow exemption from patent protection, countries which may otherwise be unable to afford certain medications, may obtain a compulsory license in emergency or lifethreatening situations. Psychotropic medications however, often may not fulfil the criteria for emergency or life-threatening treatment. The decision for governments to fund medications in low- and middle-income countries can also frequently be a difficult one, due to the lack of local evidence regarding their costeffectiveness compared with research in higher-income countries (Patel et al. 2007b; Jack et al. 2014).

General characteristics of health systems such as the existence of a comprehensive national mental health plan have been found to be associated with improved access to psychotropic medications (McBain et al. 2012). In addition the WHO has outlined the following factors in its manual for improving access to psychotropic medications: rational selection, affordable pricing, sustainable financing and reliable health and supply systems (WHO, 2005). To deconstruct the issues of access and rational use further in this paper, we focus on the medicines management cycle in the form of selection, procurement, distribution and use.

\section{Selection of psychotropic medications}

To ensure rational selection of medications where funding is limited, the WHO produces a biennial Model List of Essential Medicines (WHO, 2013). The choice of medications included is based on safety, efficacy, costeffectiveness and disease prevalence. Together the medications included should fulfil the main healthcare requirements of a population. The $\mathrm{WHO}$ also encourages the production of national lists in order to ensure that medications selected meet local needs. Almost 40 years after its introduction, at least four in five countries have adopted a national list of essential medications (WHO, 2007). However, the processes by which such lists are updated have received criticism (Barbui \& Purgato, 2014). In particular, it has been suggested that the standards of the applications for inclusion of new psychotropic medications on such lists have been relatively low. Specifically, systematic reviews of evidence are often not included in applications and conflicts of interest do not have to be declared (Barbui \& Purgato, 2014). At a local level the decision-making process behind the formation of these lists can also lack transparency (Padmanathan et al. 2014). Furthermore the extent to which national lists worldwide include psychotropic medications is unknown and updates do not always occur regularly (Raja et al. 2015). Even when the above criteria are met, psychotropic medications are generally unavailable at primary health centres and often unavailable at district-level hospitals (Padmanathan et al. 2014; Raja et al. 2015).

The choice of specific medications included in a number of essential drugs lists is also worth consideration. Diazepam appears to be frequently available at a primary care level, whereas fluoxetine (or other selective serotonin re-uptake inhibitors (SSRIs)) is often missing from lists or is only available in secondary or tertiary centres (Padmanathan et al. 2014; Raja et al. 2015; Wagenaar et al. 2015). Yet SSRIs such as fluoxetine are the first line recommended medications for depressive and anxiety disorders (Patel et al. 2007b), two conditions which make the greatest contribution to the public health burden of mental disorders worldwide (Ferrari et al. 2013).

\section{Procurement and distribution of psychotropic medications}

Effective procurement refers to obtaining the required quantity and quality of medications at the correct time at minimum cost. Factors affecting medication procurement systems include adequate funding and the type of procurement models. The proportion of budgets allocated for psychotropic medications vary between countries. However, due to the low priority often given to mental health, funding is often inadequate. In Ghana, for example, donations account for a significant proportion of funding for psychotropic medications (Raja et al. 2010).

India provides us with a good example of the outcomes resulting from different medication procurement systems due to the variability of systems between each state. A study which compared medication procurement systems in five states, found that centralised autonomous organisations were most efficient (Singh et al. 2013). The procurement process itself is also important. Pull systems in which health care facilities submit orders or estimates, are generally considered more effective at meeting local needs than push systems where standardised quantities of medications are distributed without consideration of feedback (Raja et al. 2015). Pull systems however rely on accurate documentation, which can often be hindered by a lack of technology and trained personnel. In Bihar in 
India for example, in one particular year the total consumption figures documented were five times the budgeted cost (Padmanathan et al. 2014). In contrast, in Kerala and Tamil Nadu a computer system has been used to monitor stock levels with more success (Singh et al. 2013). Furthermore, transparency and quality assurance are also vital aspects of any procurement system (Singh et al. 2013).

The lack of availability of psychotropic medications outside tertiary hospitals is often partly hindered by distribution issues. Most notably the small quantities of medications prescribed at a local or district level may not be profitable for individual companies to deliver (Padmanathan et al. 2014). This issue may be overcome with a centralised procurement and distribution system.

Small-scale Drug Resolving Funds, in which medications are sold at almost cost-price with the payment then re-invested into further medications, have been set up in areas where government funded medications are unavailable in order to improve access (Eaton, 2008). However, even if sold at cost price, such costs can often limit access to medications for the poorest, unless this is specifically addressed. In addition the small-scale nature of these projects may result in inefficiencies along the supply chain and may therefore be difficult to sustain in the long-term.

\section{Use of psychotropic medications}

Rational use of medication refers to ensuring that the correct type and dose of a drug is prescribed and taken. This should be for the correct duration, at the least cost to the community. Irrational use can have a harmful effect on both the individual and the community due to the iatrogenic and financial consequences. Numerous reasons exist for irrational use of psychotropic medications, including cultural norms, financial incentives, lack of education and inadequate regulation. Addressing these requires initiatives involving all stakeholders: patients, prescribers and regulatory bodies.

Decisions regarding who should prescribe psychotropic medications are one major issue when considering rational use. The current movement to scale up mental health care and eliminate the treatment gap encourages use of non-specialist health workers to deliver interventions. In a recent systematic review, eight publications detailed interventions in which non-specialists were trained to provide pharmacotherapy (van Ginneken et al. 2013). However in Bihar in India for example cultural norms as well as government restrictions often prevent such workers prescribing (Padmanathan et al. 2014). This was emphasised in a systematic review of the acceptability and feasibility of using non-specialist health care workers to provide mental health care; specialists expressed scepticism regarding task-sharing (Padmanathan \& De Silva, 2013). Although specialists may have genuine concerns about ensuring patient safety, a monopoly on prescribing may create conflicts of interest. It would be in a specialist's best interests to limit those able to issue prescriptions. Nonetheless several recent studies have demonstrated that it is possible to overcome the initial scepticism among specialists about the provision of mental healthcare by nonspecialists, by ensuring regular communication between all stakeholders (Padmanathan \& De Silva, 2013).

A second issue relates to how prescribing decisions should be made. Ideally, decisions would be independent and based on up to date evidence. This would rely on ensuring no financial incentives for unethical practice, such as payment per prescription written, which may lead to excessive use of repeat prescriptions. It would also rely on awareness of the limits of clinical acumen and acceptance of the important role of evidence-based guidelines (Dua et al. 2011). Although contextualisation of current guidelines is necessary, they are essential to allow transparency in decision-making. Guidelines also ensure decisions take into account up to date information regarding efficacy and cost-effectiveness, without being unduly influenced by pharmaceutical industry incentives (WHO, 2012). Furthermore they can be used to assist non-specialist health workers in prescribing.

Adherence to medications, in the form of both overand under-use, is a key issue from a patient perspective; a previous meta-analysis found that patients with mental illness often take less than half the prescribed quantity of medications (Cramer \& Rosenbeck, 1998). The reasons for this could be numerous. They include stigma associated with mental illness and its treatment, difficulty in travelling to a place where medications are accessible, the financial burden associated with buying medications, medication side effects and lack of perceived effectiveness (Pareek \& Kalia, 2013; Teferra et al. 2013). A number of practical barriers could be removed by improving access to psychotropic medications. However patient involvement in consultations is also vital to ensure correspondence between the patient and prescriber's expectations and understanding of the medications prescribed. Consequently the WHO recommends measuring consultation and dispensing times as well as patients' knowledge of the correct dosage when assessing rational use (WHO, 1993).

\section{Recommendations}

Recommendations for research in low- and middle-income countries: 
(1) Assessment of cost-effectiveness of psychotropic medications.

(2) Assessment of local psychotropic drug distribution systems using both quantitative and qualitative methods in order to identify specific areas for improvement.

(3) Local contextualisation of prescribing guidelines.

(4) Evaluation of acceptability and feasibility of prescribing psychotropic medication among trained non-specialist health workers.

(5) Assessment of patient's views regarding acceptability of and adherence to psychotropic medications.

Recommendations for policy:

(1) A local essential drugs list produced and updated regularly through a transparent, independent, evidence-based process.

(2) A pull procurement system developed with procedures to ensure careful documentation of medications dispensed to enable procurement of the correct type and quantity of medications.

(3) Funding allocated by governments specifically for essential psychotropic medications.

Recommendations for practice:

(1) Increase use of guidelines when making prescribing decisions.

(2) Ensure patient understanding of medications prescribed, likely duration required and importance of adherence.

(3) Regular monitoring of patients taking psychotropic medications, rather than indefinite repeat prescriptions without reassessment.

(4) Consequences of prescriber payment methods to be carefully considered. Payment methods should not incentivise unethical practice.

(5) Use of an active regulatory body to monitor prescribing practices.

\section{Conclusion}

Access to psychotropic medications is essential in order to address the public health burden attributable to untreated mental illnesses. In general further research, implementation of policy and changes to practice are required to address issues regarding drug selection, procurement, distribution and use. National or district-level assessment of the medications management cycles is however necessary in order to identify which, if any, of these barriers are locally relevant.

Yet it is important to once again stress that improving access to psychotropic medications should not absolve governments of responsibility for the provision of alternative interventions to treat mental illness. To reiterate a recent editorial in this journal (De Silva et al. 2015): mental health and sustainable development are deeply intertwined and therefore it is important that the broader context is not overlooked when improving access to psychotropic medications.

\section{Acknowledgement}

The authors would like to acknowledge Shoba Raja and BasicNeeds for their role in developing the ideas and concepts which informed the current work.

\section{Financial Support}

This research received no specific grant from any funding agency, commercial or not-for-profit sectors.

\section{Conflict of Interest}

None

\section{References}

Barbui C, Purgato M (2014). Decisions on WHO's essential medicines need more scrutiny. BMJ 349, g4798.

Cramer J, Rosenbeck R (1998). Compliance with medication regimens for mental and physical disorders. Psychiatric Services 48, 196-201.

De Silva M (2015). Making mental health an integral part of sustainable development: the contribution of a social determinants framework. Epidemiology and Psychiatric Sciences 24(2), 100-106.

Dua T, Barbui C, Clark N, Fleischmann A, Poznyak V, van Ommeren M, Yasamy MT, Ayuso-Mateos JL, Birbeck GL, Drummond C, Freeman M, Giannakopoulos P, Levav I, Obot IS, Omigbodun O, Patel V, Phillips M, Prince M, Rahimi-Movaghar A, Rahman A, Sander JW, Saunders JB, Servili C, Rangaswamy T, Unützer J, Ventevogel P, Vijayakumar L, Thornicroft G, Saxena S (2011).

Evidence-based guidelines for mental, neurological, and substance use disorders in low- and middle-income countries: summary of WHO recommendations. PLoS Medicine 8, e1001122.

Eaton J (2008). Ensuring access to psychotropic medication in sub-Saharan Africa. African Journal of Psychiatry 11, 179-181.

Engel GL (1977). The need for a new medical model: a challenge for biomedicine. Science 196, 129-136.

Ferrari A, Charlson F, Norman RE, Patten SB, Freedman G, Murray CJL, Vos T, Whiteford HA (2013). Burden of depressive disorders by country, sex, age, and year: findings from the global burden of disease study 2010. PLoS Medicine 10, e1001547.

Jack H, Wagner RG, Petersen I, Thom R, Newton CR, Stein A, Kahn K, Tollman S, Hofman KJ (2014). Closing the mental health treatment gap in South Africa: a review of costs and cost-effectiveness. Global Health Action 7, 23431.

McBain R, Norton DJ, Morris J, Yasamy MT, Betancourt TS (2012). The role of health systems factors in facilitating access to psychotropic medicines: a cross-sectional analysis 
of the WHO-AIMS in 63 low- and middle-income countries. PLoS Medicine 9, e1001166.

National Institute for Health and Care Excellence (2011). Common Mental Health Disorders: Identification and Pathways to Care. Manchester: NICE.

Padmanathan P, De Silva M (2013). The acceptability and feasibility of task-sharing for mental healthcare in low- and middle- income countries: a systematic review. Social Science and Medicine 97, 82-86.

Padmanathan P, Singh M, Mannarath SC, Omar M, Raja S (2014). A rapid appraisal of access to and utilisation of psychotropic medicines in Bihar, India. International Journal of Mental Health Systems 8, 29.

Pareek B, Kalia R (2013). Factors affecting non-compliance to psychotropic drugs of patients with psychosis as perceived by their family members attending the psychiatric outpatient department at selected hospital, Mangalore. Nursing and Midwifery Research Journal 9, 56-62.

Patel V (2007a). Mental health in low- and middle-income countries. British Medical Bulletin 81-82, 81-96.

Patel V, Araya R, Chatterjee S, Chisholm D, Cohen A, Silva MD, Hosman C, McGuire H, Rojas G, Ommeren M (2007b). Treatment and prevention of mental disorders in low-income and middle-income countries. Lancet 370, c991-c1005.

Raja S, Kippen Wood S, de Menil V, Mannarath SC (2010). Mapping mental health finances in Ghana, Uganda, Sri Lanka, India and Lao PDR. International Journal of Mental Health Systems 4, 1-14.

Raja S, Kippen Wood S, Reich MR (2015). Improving access to psychiatric medicines in Africa. In The culture of Mental Illness and Psychiatric Practice in Africa (ed. E Akyeampong, AG Hill and A Kleinman), pp. 262-281. Indiana University Press: Indiana.

Singh PV, Tatambhotla A, Kalvakuntla R, Chokshi (2013). Understanding public drug procurement in India: a comparative qualitative study of five Indian states. BMJ Open 3, e001987.

Teferra S, Hanlon C, Beyero T, Jacobsson L, Teshome S (2013). Perspectives on reasons for non-adherence to medication in persons with schizophrenia in Ethiopia: a qualitative study of patients, caregivers and health workers. BMC Psychiatry 13, 1-9.

van Ginneken N, Tharyan P, Lewin S, Rao GN, Meera SM, Pian J, Chandrashekar S, Patel V (2013). Non-specialist health worker interventions for the care of mental, neurological and substance-abuse disorders in low- and middle-income countries. Cochrane Database of Systematic Reviews 11, CD009149.

Wagenaar BH, Stergachis A, Rao D, Hoek R, Cumbe V, Napú M, Sherr K (2015). The availability of essential medicines for mental healthcare in Sofala, Mozambique. Global Health Action 8, 27942.

Whiteford HA, Degenhardt L, Rehm J, Baxter AJ, Ferrari AJ, Erskine HE, Charlson FJ, Norman RE, Flaxman AD, Johns N, Burstein R, Murray CJL, Vos T (2013). Global burden of disease attributable to mental and substance use disorders: findings from the Global Burden of Disease Study 2010. Lancet 382, 1575-1586.

World Health Organisation (1993). How to Investigate Drug use in Health Facilities. Selected drug use indicators. EDM Research Series No.7. World Health Organization: Geneva. WHO/DAP/93.1.

World Health Organisation (2001). The World Health Report: Mental Health: New Understanding, New Hope. World Health Organisation: Geneva.

World Health Organisation (2005). Improving access and use of psychotropic medicines. In Mental Health Policy and Service Guidance Package (ed. M Everard, H Hogerzeil, M Funk, B Saraceno and C Lund), pp. 1-74. World Health Organisation: Geneva.

World Health Organisation (2007). The WHO Essential Medicines list: 30th Anniversary. World Health Organisation: Geneva. Retrieved 7 June 2015 from http://www.who.int/ medicines/events/fs/en/.

World Health Organisation (2011a). mhGAP Intervention Guide for Mental, Neurological and Substance use Disorders in Non-Specialized Health Settings. World Health Organisation: Geneva.

World Health Organisation (2011b). Access to essential medicines as part of the right to health. In The World Medicines Situation, 3rd edn. (ed. A Cameron, M Ewen, M Auton and D Abegunde), pp. 1-12. World Health Organisation: Geneva.

World Health Organisation (2012). The Pursuit of Responsible Use of Medicines: Sharing and Learning from Country Experiences. World Health Organisation: Geneva.

World Health Organisation (2013). WHO Model List of Essential Medicines, 18th list. World Health Organisation: Geneva. 